\title{
Three-Dimensional Joit Kinematics Using Bone Surface Registration: A Computer Assisted Approach with an Application to the Wrist Joint in vivo
}

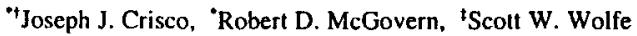 \\ 'Department of Orthopaedics, Rhode Island Hospital, Providence. RI; \\ 'Division of Engineering. Brown University, Providence, RI; \\ 'Department of Orthopaedics, Yale University School of Medicine, New Haven, CT
}

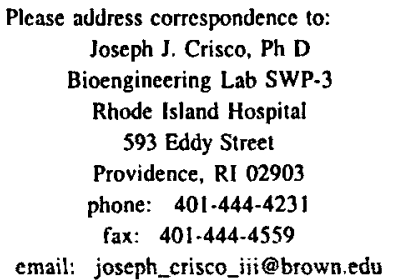

\begin{abstract}
The majority of the present knowledge of the three-dimensional kinematic behavior of skeletal joints has been acquired in studics employing cadaveric models using invasive procedures. In the wrist, the small size and complex motion of the carpal bones present a difficult challenge for an invasive kinematic analysis or external marker system. This paper describes an approach to quantify the three-dimensional kincmatics of the wrist and carpal bones in vivo, using non-invasive computed tomographic (CT) imaging. The applications of this method include quantification of normal wrist motion, analysis of pathomechanics, and evaluation of surgieal intervention. The approach is also applicable to other joints and imaging modalities.
\end{abstract}

\section{INTRODUCTION}

Kinematic analysis has been widely used to study normal joints, and the effects of pathology, acute trauma, and reconstructive procedures on joint motion. Most of the studies on human joints have been performed in cadaveric specimens. Previous in vivo joint motion studies have been limited to a two-dimensional kinematic analysis with plane radiography or to invasive procedures such as transcutaneous bone pins' or implantable bone markers'. Such invasive procedures are not well accepted by volunteers and the utility of bone pins is further compromised by mechanical impingement and tethering of soft tissues. The advantages of analyzing joint motion in vivo include the incorporation of true muscular forces, which are either neglected or simulated in cadaveric studies, and the ability to evaluate the long term effects of healing and surgical intervention. Recently, researchers have successfully measured the three-dimensional in vivo kinematics of the long bones of the knee joint using noninvasive techniques'. Due to the complexity, shape and small size of the carpal bones, the existing techniques for measuring three-dimensional kinematics are not applicable to the wrist.

Our aim was to develop a noninvasive method to measure 3D in vivo motion of the carpal bones. The purpose of this paper is to briefly present our approach and interactive computer method for quantification and visualization of complex 3D in vivo kinematics. This method may be used to evaluate surgical treatments, study kinematics of joint replacement, generate quantified diagnostic information on aberrant kinematics, and provide 3D animation for professional and patient education.

\section{METHODS}

For the purposes of illustrating the method, the in vivo kinematics of selected carpal bones were quantified in a single uninjured subject. The steps described in the method are image acquisition, segmentation of bone surfaces, kinematic analysis, and visualization. 
Image acquisition was performed while the left wrist was positioned in three different positions: radial deviation, neutral, and ulnar deviation. Volume images of a $60 \mathrm{~mm}$ segment of the wrist extending from the radioulnar metaphysis to the proximal metacarpals were acquired using a HiSpeed Advantage CT scanner (GE Medical Systems, Milwaukee, WI) and exposure parameters of $80 \mathrm{~mA}, 80 \mathrm{kVp}$ and 1 second. Volume images were collected in axial format with voxel dimensions of $0.187 \times 0.187 \times 1 \mathrm{~mm}^{3}$. The images were transferred to a Silicon Graphics workstation (Indigo ${ }^{2} \mathrm{XZ}, \mathrm{SGI}$, Mountain View, CA) on which all image processing was done using a 3D biomedical imaging software system (Analyze, Biomedical lmaging Resource, Rochester, MN).

Segmentation was defined as the process of extracting bone contours and associating each contour with the individual carpal bones. Defining the outer surfaces of the eight carpal bones presents a challenging segmentation problem because of the narrow articular spaces and highly curved shapes. Segmentation was performed in several steps: thresholding, extraction, and association. The CT volumes were thresholded to emphasize the outer cortical shell. In each image slice, bone contour coordinates were extracted after each bone cross-section was closed and filled ${ }^{\text {to }}$. This resulted in multiple contours for each cross-section. To associate each contour with its respective carpal bone, we developed an interactive approach using custom software (Open Inventor, C+t, Silicon Graphics, Inc; MATLAB, Mathworks, Natick, MA). The software generated 3D images of the contours and created an interactive handle at each contour's centroid. The interactive handles enabled the user to manipulate the view and manually associate each contour with its proper bone. Segmentation was completed by exporting the 3D surface coordinates for the contours of each bone.

Kinematic analysis was performed under the assumption that each bone moved independently as a rigid body in 3D. The kinematic variables were calculated by registering the bone, described by its surface points obtained from segmentation, from one position to another position. Registering the bone's centroid and principal axes of inertia provided an initial estimate of the kinematic variables ${ }^{3}$. Final registration and the resulting kinematic variables were obtained by minimizing the mean distance between the bone's surfaces using an algorithm developed by Pelizzari et $\mathrm{al}^{9}$. The helical axis of motion (HAM) was chosen to uniquely describe the $3 \mathrm{D}$ kinematics with a rotation about and translation along an axis in space. The motions of each of the capitate, scaphoid, and lunate bones were calculated relative to the radius.

Vistualization of bone position and orientation was helpful to ensure successful reconstruction of the images. The segmented contours were rendered as solid bone cross-sections using specifically written code in $\mathrm{C}++$ and Open Inventor. Better visualization and an improved estimate of the bone's true surface was obtained by generating a triangular surface mesh (B. Geiger, Nuages, INRIA, France). Animations using the kinematic data and reconstructed bones can also be created to provide dynamic viewing of the results.

\section{RESULTS}

The simple motion of wrist radial and ulnar deviation resulted in complex coupled motions of the cappal bones that were visualized by rendering the bone surfaces. These renderings are three dimensional models that can be viewed from any orientation (Fig. 1).

For the single subject in radial deviation, the motions of the scaphoid and lunate were notably distinct from those of the capitate (Fig. 2A). The helical rotation angle of the capitate (33.9') was nearly twice that of both the scaphoid $\left(18.4^{\circ}\right)$ and lunate $\left(14.7^{\circ}\right)$. The helical translation of the capitate $(0.7 \mathrm{~mm})$ was similar to that of the scaphoid $(0.6 \mathrm{~mm})$, while there was no measurable helical translation of the lunate $(<0.01 \mathrm{~mm})$. Each helical axis passed through the proximal portion of the capitate. The orientation of the helical axis of the capitite was approximately dorsal-ventral, while the helical axes of the scaphoid and lunate were roughly orthogonal in a mediallateral orientation. These relative orientations of the helical axis demonstrate that the scaphoid and lunate exhibited coupled flexion as the capitate rotated with the wrist in radial deviation.

In ulnar deviation, the motions of scaphoid and lunate were more similar to those of the capitate (Fig. 2B). The helical rotation of the capitate $\left(25.9^{\circ}\right)$ was similar to both the scaphoid $\left(25.2^{\circ}\right)$ and lunate $\left(24.8^{\circ}\right)$. In contrast to radial deviation, the helical translations of each carpal bone were notable and similar (each approximately $2.5 \mathrm{~mm}$ ). While the helical axes passed through the proximal capitate, as in radial deviation, there was less of a difference in their orientation.

\section{DISCUSSION}

We have demonstrated a method to accurately and noninvasively measure 3D joint kinematics in vivo. The method was illustrated using CT volume images of a single wrist, however it can be applied to the study of all skeletal joints with any medical imaging modality from which 3D surfaces can be accurately segmented. Despite the small size, complex shape and motion patterns of the carpal bones, the present method was shown to be capable of tracking in vivo 3D motions. The motions reported here were similat to those reported previously using cadaveric specimens implanted with radio-opaque markers ${ }^{6}$. A more detailed comparison can be accomplished orice this methodology has been a applied to a larger number of subjects. 
A detailed analysis of accuracy is an extensive study because of the numerous contributing factors such as bone size and shape, voxel size, image quality, scanning orientation, and the particular registration method. Accuracy varies for each bone within the same the wrist image because of differences in bone size and shape. A preliminary study of kinematic accuracy found that the HAM angle and translation errors were typically less than 0.6 degrees and $0.5 \mathrm{~mm}$, although the lunate errors were slightly larger ${ }^{4}$. When the method is employed to study pathokinematics, the most crucial issue will become the level of accuracy required to detect clinically significant differences, and this will be determined in part by the specific clinical disease to be studied. Each of these factors requires further study.

Application of segmentation techniques to medical imaging has developed rapidly and several investigators have developed protocols for use in studying the carpus ${ }^{2}, 8,13$. A detailed comparison of the efficacy of the various approaches to segmenting the bone surface is difficult because of the lack of a common data base, however, any scymentation method may be used with the kinematic analysis presented here. One limitation of our method is that the calculated kinematics are quasi-static; motion is calculated between two fixed positions. This limitation is not inherent in the method, but is due to the time required to acquire multiple CT volume images. Decreasing the interval between fixed positions will improve the ability of this technique to simulate true motion, however radiation exposure will increase. Future advances in real time scanning techniques may eliminate this limitation. While radiation exposure is always a concern, the recent generation CT scanner used in this study produced exposure levels for nine complete volume images that totaled well below governmental guidelines.

The complexity and the number of wrist bones results in a voluminous amount of kinematic data. Our approach includes a method for visualizing these complex motions which should help in understanding joint kinematics and aid in research and educational applications. Future studies should also generate a database with which in vitro and computer models can be validated. The application of this 3D kinematic method to pathological conditions and to evaluation of surgical interventions may assist in diagnosis and treatment of complex disorders of the carpus.

\section{ACKNOWLEDGMENTS.}

We would like to thank Lee Katz, M.D. for his assistance in acquiring the CT images. This work was supported in part by NIH AR44005.

\section{REFERENCES}

1. Banks SA, Markovitch GD. Hodge WA. In vivo kinematics of the cruciate retaining and substituting knee replacements. $J$ Arthroplasty, 12(3): 297-304, 1997.

2. Belsole RJ, Hilbelink DR, Llewellyn JA, Dale M, Ogden JA. Carpal orientation from computed reference axes. $J$ Hand Surg. 16A(1): 82-90, 1991.

3. Crisco JJ, McGovern RD. Efficient calculation of mass moments of inertia for segmented homogeneous 3D objects. $J$ Biomech, 31(1):97-101, 1998.

4. Crisco JJ, McGovern RD, Wolfe SW. Three dimensional kinematics of carpal bones: an in vitro error analysis. Am Soc Biomech Conference, Clemson, SC, Sept 22-24, 1997.

5. de Lange A, Kauer JMG, Huiskes R. Kinematic Behavior of the Human Wrist Joint: A Roentgen-Stereophotogrammetric Analysis. J Orthop Res, 3:56-64, 1985.

6. Horii E, Garcia-Elias M. An KN, Linscheid RL, Bishop AT, Cooney WP, Chao EYS. A kinematic study of lunotriquetral dissociations. J Hand Surgery, 16(2):355-362, 1991.

7. Lafortune MA, Cavanagh PR. Sommer HJ, Kalenak A. Three-dimensional kinematics of the human knee during walking. $J$ Biomech, 25(4):347-358, 1992.

8. Patterson RM, Elder KW, Viegas SF, Buford WL. Carpal bone anatomy measured by computer analysis of threedimensional reconstructions of computed tomography images. J Hand Surgery, 20(6):923-929, 1995.

9. Pelizzari CA, Chen GT, Spelbring DR, Weichselbaum RR, Chen CT. Accurate three-dimensional registration of CT, PET, and/or MR images of the brain. J Comput Assist Tomogr, 13(1):20-26, 1989.

10. Robb RA. Three-dimensional Biomedical Imaging: Principles and Practice. VCH, N.Y., N.Y., 1995.

11. Viegas SF, Hillman GR, Elder K, Stoner D, Patterson RM. Measurement of carpal bone geometry by computer analysis of three-dimensional CT images. J lland Surg. 18A(2):341-349, 1993. 


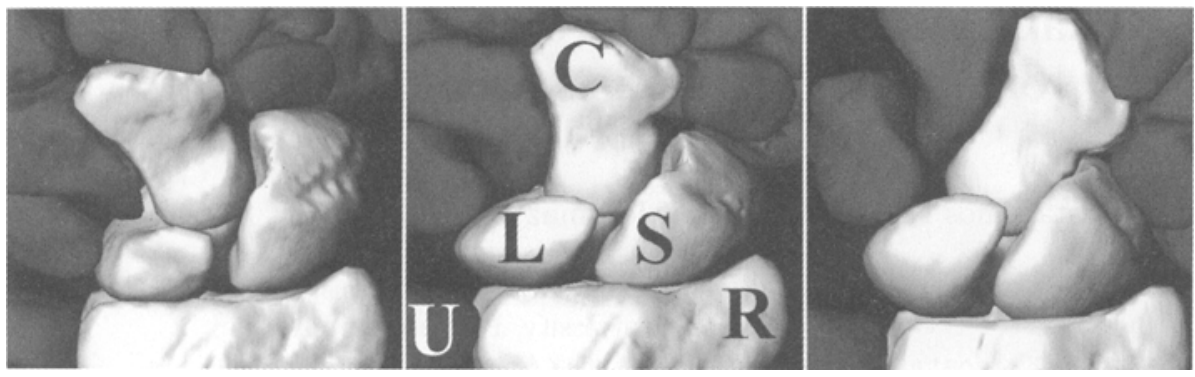

Fig. 1. Three-dimensional dorsal view of the left wrist of a healthy subject. The CT volume images were collecied in ulnar deviation (left), neutral (middle) and radial deviation (right) of the wrist. The long axis of the capitate (C) follows wrist motion which is occurring in the plane of the paper. These renderings illustrate the complex 30 motion of the scaphoid (S) and lunate (L): in uinar deviation the lunate and the scaphoid also extend (rotate out of the paper) and in radial deviation they also flex (totatc into the paper), relative to their ncutral orientations. For reference and clarity the ulna (U) and radius (R) are labeled and the other bones have been darkened.

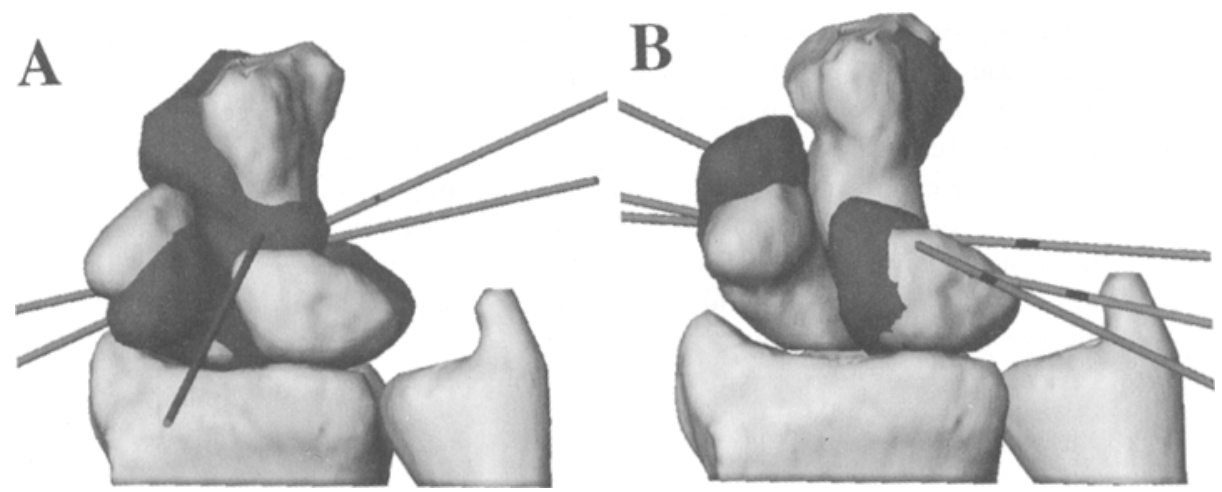

Fig. 2. The location and orientation of the helical axes are rendered here for the motion of the scaphoid, lunate and capitate from the neutral position to radial deviation $(A)$ and ulnar deviation (B) relative to the radius (palmer view). 